First publ. in: Brain Topography 7 (1995), 4, pp. 291-299

\title{
Neuromagnetic Source Analysis Using Magnetic Resonance Images for the Construction of Source and Volume Conductor Model
}

\author{
Bernd Lütkenhöner*, Elisabeth Menninghaus*, Olaf Steinsträter*, Christian Wienbruch*, Hans \\ Martin GiBler ${ }^{+}$, and Thomas Elbert*
}

\begin{abstract}
Summary: Sources of the somatosensory evoked fields (SEF) for one subject were estimated using constraints from the magnetic resonance images (MRI) of the same subject. A realistic volume conductor model was shaped corresponding to the inside of the skull. Sources were restricted to a dipole patch riding on the surface of the cortex, reconstructed from the individual MRI. Such a patch can be considered as a uniformly activated cortical area giving rise to distributed currents which flow perpendicular to the cortical surface. Source locations obtained for the SEF in response to separate stimulations of lower lip, first and fifth digit, and collarbone followed the course of the contralateral central sulcus. The order of the estimated source locations was in agreement with the somatosensory homunculus of Penfield and Rasmussen. Similar results were obtained with the simple model of a current dipole in a homogeneous sphere. In contrast, combining a current dipole model with a realistic volume conductor model was rather problematic as it overestimates the radial dipole component by an order of magnitude.
\end{abstract}

Keywords: Biomagnetism; Source analysis; Anatomical constraints; Current dipole; Dipole patch model; Somatosensory evoked fields.

\section{Introduction}

Model simulations (Hari et al. 1988; Kuriki et al. 1989) as well as phantom measurements (Menninghaus et al. 1994) suggest that for magnetic fields generated by a single dipolar source, the source localization accuracy is of the order of a few millimeters, provided that the signal-to-noise ratio of the biomagnetic data is sufficient and that an appropriate volume conductor model is used. In the case of two or more simultaneously active sources such a high accuracy can be expected only if the distances between the sources are sufficiently large. It is clear that a reliable source separation becomes increasingly difficult with decreasing inter-source distances so that for distances below a certain limit (the critical inter-source distance) the sources are indistinguishable from one single equivalent source. This transition is of course fluid, owing to superimposed noise in the measured data,

*Institute for Experimental Audiology, Münster, Germany.

'Radiological Clinic, University of Münster, Münster Germany.

Accepted for publication: January 25, 1995.

This work was supported by a grant from the Deutsche Forschungsgemeinschaft (Klinische Forschergruppe 'Biomagnetismus und Biosignalanalyse').

Correspondence and reprint requests should be addressed to Dr. B. Lütkenhöner, Institute for Experimental Audiology, Kardinal-vonGalen-Ring 10, D-48129 Münster, Germany. so that the critical inter-source distance must be understood in a statistical sense (e.g., defined as the distance guaranteeing a correct source separation in at least $50 \%$ of the cases). Since the critical inter-source distance is dependent on numerous factors (e.g., signal-to-noise ratio, depth of the sources, orientations of the dipole moments), it is impossible to give a general characterization of the conditions on which a reliable source separation can be expected. However, a rule of thumb which has proven to be consistent with numerous model investigations (e.g., Lehnertz et al. 1989; Tan et al. 1990; Lütkenhöner 1991, 1992a,b, 1994a) as well as with experimental data (Lütkenhöner et al. 1992; Lütkenhöner and Pantev 1992) can provide a rough idea of the source separability: the critical inter-source distance is generally of the same order of magnitude as the distances between sources and measurement coils.

Since the distances between measurement coils and sources are generally $5 \mathrm{~cm}$ or greater in neuromagnetic studies, the above rule implies that neuromagnetic source analysis is a technique with low spatial resolution. Fortunately, the rule is valid only for the case that a source estimation is based exclusively on magnetic field measurements (unconstrained inverse problem). A different situation may arise if the set of possible solutions (parameter space) can be reduced by making appropriate assumptions or by providing additional information. In many neuromagnetic studies it is reasonable to assume 
that the observed field pattern is produced by current sources located on the cortical surface. Model simulations with a simple model of a sulcus of the brain (Lütkenhöner 1994b) have already shown that this type of information can improve the localization accuracy significantly.

Therefore it is obvious that the usefulness of biomagnetic source analysis is highly dependent on the development of more advanced techniques. A natural step in this direction would be the integration of anatomical information from magnetic resonance images (MRI). Previously, MRI data were only used to establish the plausibility of dipole locations within an anatomical context (e.g., Kuriki et al. 1988; Reite et al. 1988; Yamamoto et al. 1988; Pantev et al. 1990). The strategy for the future, however, must be to extract anatomical information from the MRI prior to the source analysis and to tailor both the source and the volume conductor model accordingly.

As far as the volume conductor model is concerned, replacement of the standard model of a homogeneous sphere by a homogeneous volume with a surface corresponding to the inner surface of the skull (or an envelope of the brain) would already represent a significant step forward. The numerical efforts required for dealing with this type of realistic volume conductor model are relatively low since only the surface of the volume conductor has to be considered and efficient algorithms (boundary element method) are available (Meijs et al. 1987; Hämäläinen and Sarvas 1989; Menninghaus et al. 1994).

One prerequisite for a realistic source model is the availability of a reconstruction of the cortical surface. It shall be assumed here that sources not located on the cortical surface provide negligible contributions to the measured magnetic field. Though this assumption appears reasonable for many experimental conditions, some care is required since situations are conceivable where this assumption is inappropriate. Algorithms suitable to extract these surfaces from MRI data were described by Dale and Sereno (1993) as well as Fuchs et al. (1994).

We intend to analyze an actual MRI based model in terms of its workability, to demonstrate the serious errors that may result from a too straightforward implementation, and to present possible solutions to these problems.

\section{Methods}

\section{Neuromagnetic Experiments}

Data were taken from an ongoing study of somatosensory evoked fields (SEF). Since the article mainly deals with strategies for combining MEG and MRI rather than $\mathrm{SEF}$, only one subject was studied. The stimuli consisted

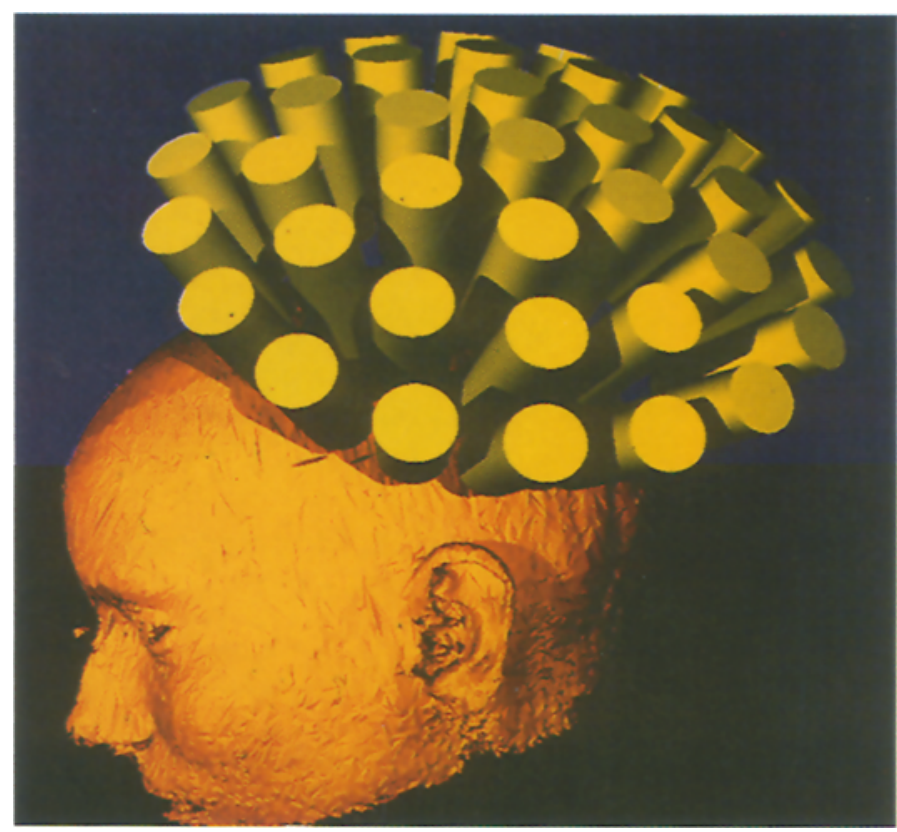

Figure 1. Ray-traced image of scalp surface (reconstructed from MRI), and 37-channel neuromagnetometer.

of slight pressure pulses which were applied via a pneumatic stimulator using a standard non-painful stimulus intensity. Four sites were stimulated: the lower lip near the right corner of the mouth, the right first and fifth digit, and the right collarbone. For each stimulus condition, 1000 stimuli were delivered with a mean interstimulus interval of $500 \mathrm{~ms}$. The magnetic field was recorded in a magnetically shielded room with a 37-channel gradiometer system (Biomagnetic Technologies, San Diego) centered over position $\mathrm{C} 3$ of the international 10-20 system for electrode placement (figure 1 ). The data were bandpass filtered between $0.1 \mathrm{~Hz}$ and $30 \mathrm{~Hz}$, sampled at $521 \mathrm{~Hz}$, and averaged after artifact rejection with a threshold of $2 \mathrm{pT}$ peak-to-peak. The number of averaged epochs ranged between 680 and 870 . For each of the four investigated data sets, the time slice analyzed corresponded to the highest root-mean square value within the first $100 \mathrm{~ms}$ after stimulus onset. These times were located in the interval between $52 \mathrm{~ms}$ and $88 \mathrm{~ms}$.

\section{MRI and Surface Reconstructions}

MRI data were acquired using a $1.5 \mathrm{~T}$ Magnetom (Siemens, Erlangen, Germany). To fulfill the demand for a fast imaging sequence, short measurement time, and good contrast of grey and white matter, a rapid T1 weighted 3D gradient echo sequence (MPRAGE-T14B195) was used: $180^{\circ}$ inversion pulse as the preparation pulse, TI $300 \mathrm{~ms}$, TR $10 \mathrm{~ms}$, TE $4 \mathrm{~ms}$, field of view $256 \mathrm{~mm}$, flip angle $10^{\circ}$, thickness of the 3D slab $192 \mathrm{~mm}$, effective 


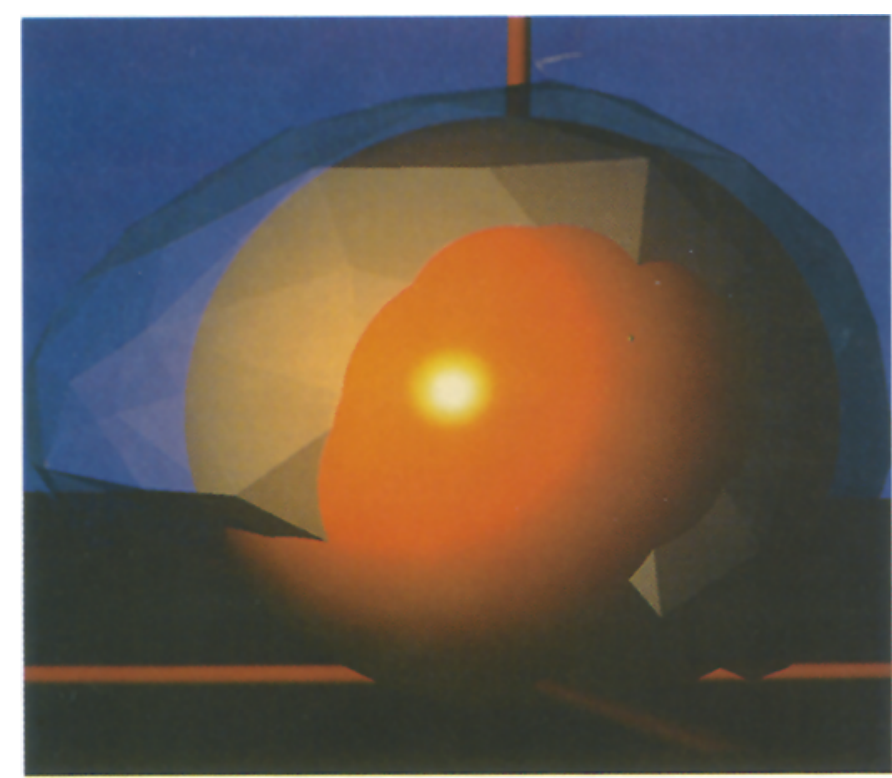

Figure 2. Comparison between realistic volume conducfor model and best-fitting sphere. The outlines of the sphere are visible also under the surface of the realistic volume conductor model, because the latter was plotted as semi-transparent. The surface of the sphere, on the other hand, was assumed to be opaque so that the surface of the realistic volume conductor model is partially hidden.

slice thickness $1.5 \mathrm{~mm}$. Three landmarks filled with gadolinium DTPA solution were used for coordinate system matching. These were placed at the preauricular points and the nasion.

The surface of the volume conductor model was constructed in two steps. First, rough 2D contours of the brain were extracted successively from each of the 128 MRI slices using an interactive segmentation software. A triangulated 3D surface was then generated. The resulting surface, visualized in figure 2 as a transparent shell, is composed of 380 plane triangles (192 vertex points).

For the surface reconstruction of the cortex a newly developed 3D algorithm (Steinsträter 1994) was used. In the first step (rough segmentation), three different sets of voxels are distinguished: those which can be attributed irrevocably to the brain (brain voxels) or its complement (non-brain voxels), and those for which no clear assignment to one of the first two sets of voxels can be achieved (remaining voxels). This first step is subdivided into three substeps: preprocessing (elimination of artifacts), definition of the white matter (brain voxels) with a floodfilling procedure exploiting the relatively high contrast between gray and white matter, and use of a distance transformation to determine the non-brain voxels. In the second step, the remaining voxels are assigned to either the brain or the non-brain voxels using a concurrent growing process. The result is a binary matrix where all brain voxels have the value 1 and all non-brain voxels have the value zero. In the third step, the surface of the brain is triangulated and smoothed using an algorithm similar to that described by Dale and Sereno (1993). In the example presented here, the cortical surface was represented by about 800,000 triangles (with about 400,000 vertex points).

\section{Unconstrained source analysis}

Source analyses using the model of a current dipole in a homogeneous sphere were performed as described in Lütkenhöner et al. (1991) and Lütkenhöner (1992b). Briefly, the original least-squares fit problem was transformed into a minimization problem for the non-linear parameters (dipole coordinates) by replacing the linear parameters (components of the dipole moment) with the algebraic solutions available for their least-squares estimates. The resulting minimization problem was solved iteratively by means of Powell's method (Press et al. 1992). Starting values for the iteration procedure were obtained by an exhaustive search in a three-dimensional grid ( $5 \mathrm{~mm}$ spacing between adjacent gridlines).

The center of the sphere was estimated by means of a least-squares fit algorithm (Lütkenhöner et al. 1990), taking into account only that part of the volume conductor which was located below the 37 measurement coils. Figure 2 illustrates the good agreement between the realistic volume conductor and the best-fitting sphere. Fitting the surface of the scalp rather than the surface of the realistic volume conductor model resulted in practically the same center of sphere.

Parameter estimations with the realistic volume conductor model were carried out in the same way as with the spherical model, except that some calculations were delegated to a separate program. This auxiliary program provided a data file with the leadfield vectors for all dipole positions in a regular three-dimensional grid (5 $\mathrm{mm}$ spacing between adjacent grid lines). Leadfield calculations were carried out using the boundary element method as described in Menninghaus et al. (1994). To deal with arbitrary dipole positions, the dipole fit program performed a trilinear interpolation on a grid cube (analogous to the bilinear interpolation on a grid square described in Press et al. 1992).

\section{Source analysis with anatomical constraints}

Parameter space constraints were introduced by assuming that the magnetic field is produced by currents flowing perpendicular to the surface of the cortex. The model of a single current dipole was replaced for that purpose by a dipole patch model. Figure 3 shows this 


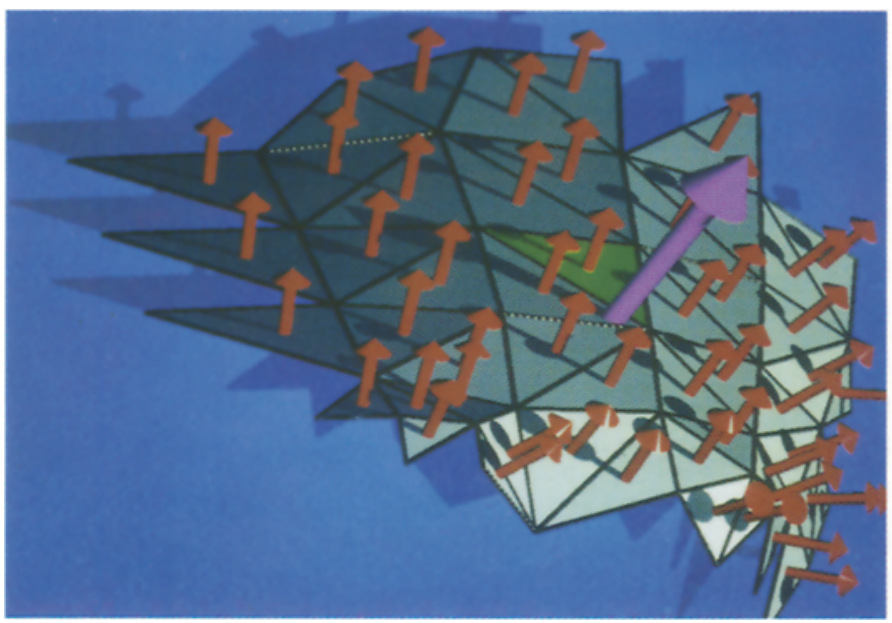

Figure 3. Dipole patch model. The patch shown in this figure has an area of $10 \mathrm{~mm}^{2}$ and is represented by 51 triangles. The green triangle served as the starting point ("seed") for the patch construction. Each triangle is used as a platform for a current dipole oriented perpendicular to the surface (arrows displayed in red, located in the centers of the triangles). The huge magenta arrow can be interpreted as the equivalent current dipole representing the totality of the dipoles on the patch. It points into the same direction as the vectorial sum of the moments of the red dipoles, and its location corresponds to the mean location of the red dipoles.

model in detail, whereas figure 4 shows the model and the surrounding cortical surface in a reduced scale. The idea underlying the dipole patch model is that the source of the magnetic field corresponds to a uniformly activated patch of the cortical surface. The implementation of the dipole patch model, as shown in figure 3 and figure 4 , exploits the fact that the cortical surface is available in a discretized form. To model a uniform activation, each of the triangles forming the patch is provided with an outside-pointing dipole perpendicular to the surface. The dipole locations coincide with the centers of the triangles. Identical amplitudes are chosen for the moments of all dipoles. (If the areas of the triangles would largely differ, it would be advisable to choose the dipole moments proportional to the surface of the respective triangle.)

The construction of a patch starts with a single triangle $\left({ }^{u}\right.$ seed $\left.^{\prime \prime}\right)$, displayed in green in figure 3 . Then, in an iterative procedure, triangles are added until the area of the resulting patch exceeds a pre-defined value $\left(10 \mathrm{~mm}^{2}\right.$ in the present study). The algorithm selects the triangles such that the resulting patch is as compact as possible. For this reason, each triangle of the patch is provided with a number measuring the cumulative distance to the starting triangle. The calculation of the cumulative distances is initiated by defining the cumulative distance of

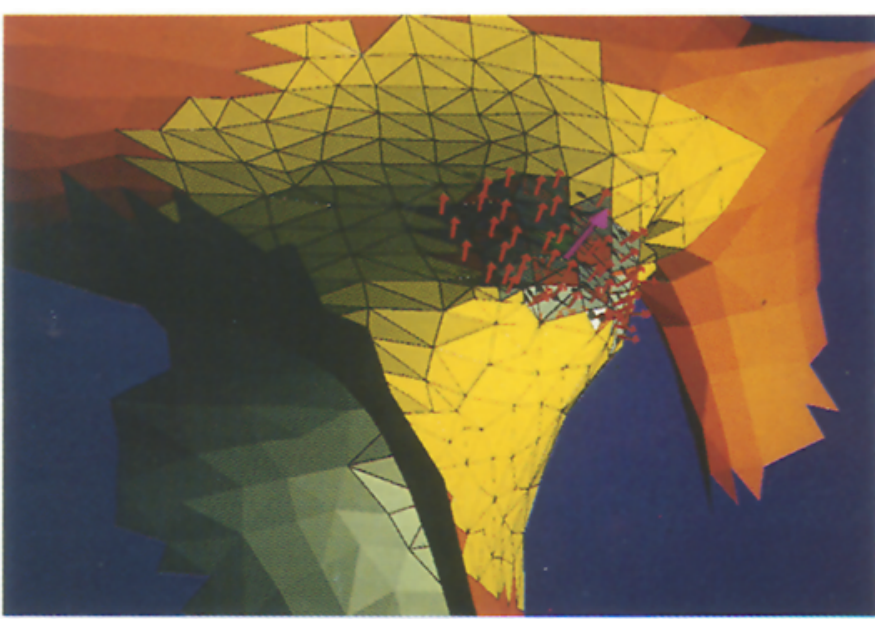

Figure 4. Dipole patch from figure 3 and its vicinity. The yellow surface represent an extended patch with an area of $1 \mathrm{~cm}^{2}$ (dipole symbols omitted), and the orange area ( $\pi$ $\mathrm{cm}^{2}$, partially clipped) shows the adjacent cortical surface. The reverse of reconstructed surface was given a grey pigment. It can be deduced from this figure that location and orientation of the equivalent current dipole associated with a patch is, in general, dependent on the area of the patch.

the starting triangle as zero. In any of the following steps, the cumulative distances are evaluated for all triangles sharing at least one side with one of the triangles already assigned to the patch, and the triangle with the smallest cumulative distance is added to the patch.

Except for the starting triangle, the cumulative distance of a triangle is easily derived from the cumulative distances of its neighbors. Suppose that triangle $T$ shares one side with a triangle $P$ already belonging to the patch. If $\Delta_{P}$ denotes the cumulative distance assigned to triangle $P$, and $\delta_{T, P}$ denotes the distance between the centers of the triangles $T$ and $P$, then the cumulative distance of triangle $\mathrm{T}$ (when accessed via triangle $\mathrm{P}$ ) will be $\Delta_{P}+\delta_{T, P}$. If triangle $\mathrm{T}$ can also be accessed via a second triangle belonging to the patch, then the cumulative distance will also be calculated for the second access possibility, and the optimal access possibility will be selected.

It is obvious that the dipole patch model can be used in way similar to the model of a single current dipole. An important difference, however, is that the number of parameters is halved: There are only two location parameters and one single amplitude parameter. To find the optimal source location on the surface of the cortex it is convenient to exploit the fact that, in the present case, this surface is available in a discretized form, represented by a finite number of plane triangles. Thus, by considering the centers of the these triangles as the possible source 


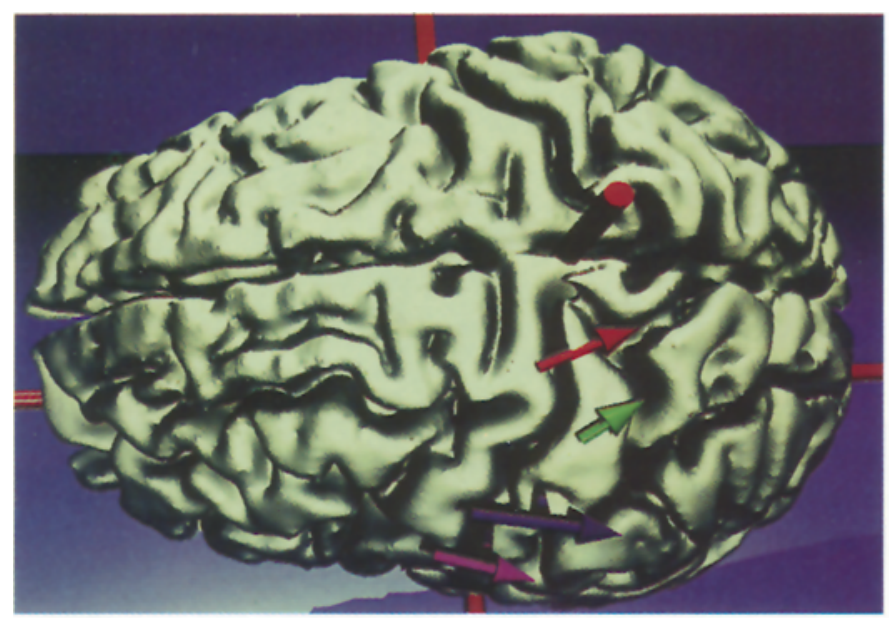

Figure 5. Reconstruction of the cortical surface with dipoles estimated from somatosensory evoked fields. The source analysis was performed using the model of a current dipole in a spherical volume conductor. The locations of the four dipoles (magenta representing the lip. blue the first digit, green the fifth digit, and red the collarbone) follow the central sulcus. Arrow heads corresponding to $5 \mathrm{nAm}$.

locations (i.e. the centers of the dipole patches), the search for the optimal source location can be reduced to a search for the "optimal triangle".

It is clear that strategies for selecting a subset of triangles had to be devised since the number of triangles was quite high in the present study. Two principal possibilities can be distinguished. First, the search can be confined to those triangles which are sufficiently close to the expected dipole location. Second, a certain subset of triangles can be selected randomly. Both possibilities were exploited in the present study to obtain a rough first estimate of the optimal dipole location. Then, in a subsequent step, a refined estimate was obtained by considering all triangles within a distance of $2 \mathrm{~cm}$ from the rough estimate.

\section{$3 D$ visualization}

The surfaces reconstructed from the MRI as well as the dipoles estimated from the biomagnetic data were put into a virtual three-dimensional world. Photo-realistic images from this virtual world were taken using the ray-tracing software POVRAY 2.2, a copyrighted freeware available on the Internet (see also Young and Wells 1994). This software is operated via a scene description language which allows a flexible definition of camera, light sources, and objects (specified in terms of shape, color, texture).

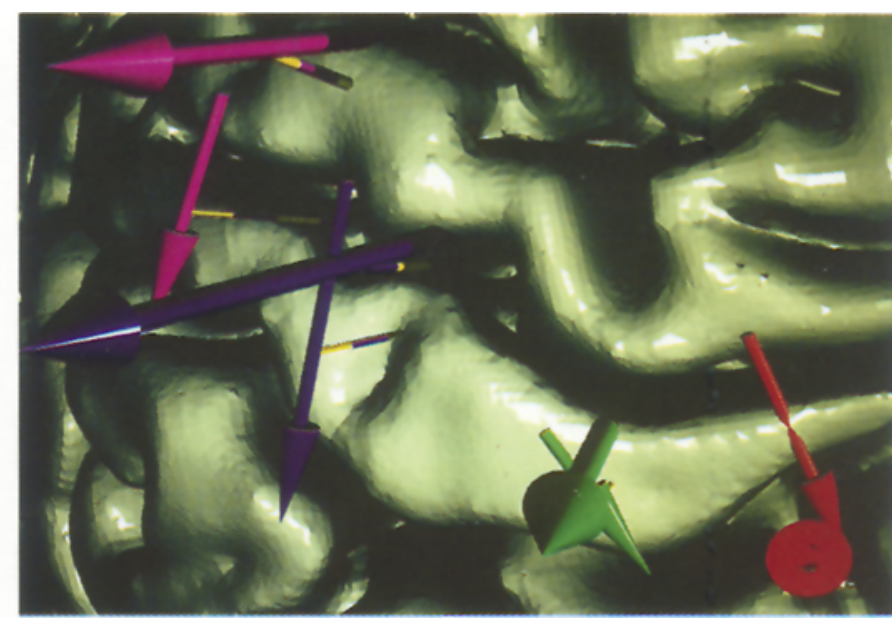

Figure 6. Detailed view of the central sulcus and its vicinity with fit results provided by the model of a current dipole in a realistic volume conductor (arrow heads corresponding to $10 \mathrm{nAm}$ ). For comparison, the small dipoles show the results obtained with the spherical model as plotted already in figure 5. All the dipoles were shifted outwards by $2 \mathrm{~cm}$. The attached scales are subdivided in steps of $5 \mathrm{~mm}$.

\section{Results}

\section{Unconstrained source analysis}

Results obtained with the model of a single current dipole in a spherical volume conductor shall be considered first. Figure 5 shows the dipoles estimated for the four different stimuli in their anatomical context. Because the estimated dipoles are located inside the brain, and therefore hidden by the cortical surface, dipole symbols were radially shifted to the outside $(2 \mathrm{~cm})$. Figure 5 shows that the estimated dipole locations follow the course of the central sulcus. The order of the dipoles is in agreement with the somatosensory homunculus of Penfield and Rasmussen (1950): Most laterally, the lip (magenta); then the first digit (blue) and the fifth digit (green); and finally, most medially, the collarbone (red). The goodness of fit values were $98.5 \%$ (lip), $96.5 \%$ (first digit), $99.2 \%$ (fifth digit), and $95.1 \%$ (collarbone).

Figure 6 shows a more detailed view of the central sulcus and its vicinity. Small arrows correspond to the same dipoles as in figure 5; large arrows represent the dipoles estimated after having replaced the spherical model with the realistic volume conductor model. Because larger dipole amplitudes were obtained in the latter case, arrow lengths were reduced by a factor of four compared to the small arrows. Goodness of fit values obtained in the latter case were $99.6 \%$ for the lip, $98.9 \%$ for the first digit, $98.8 \%$ for the fifth digit, and $94.7 \%$ for 


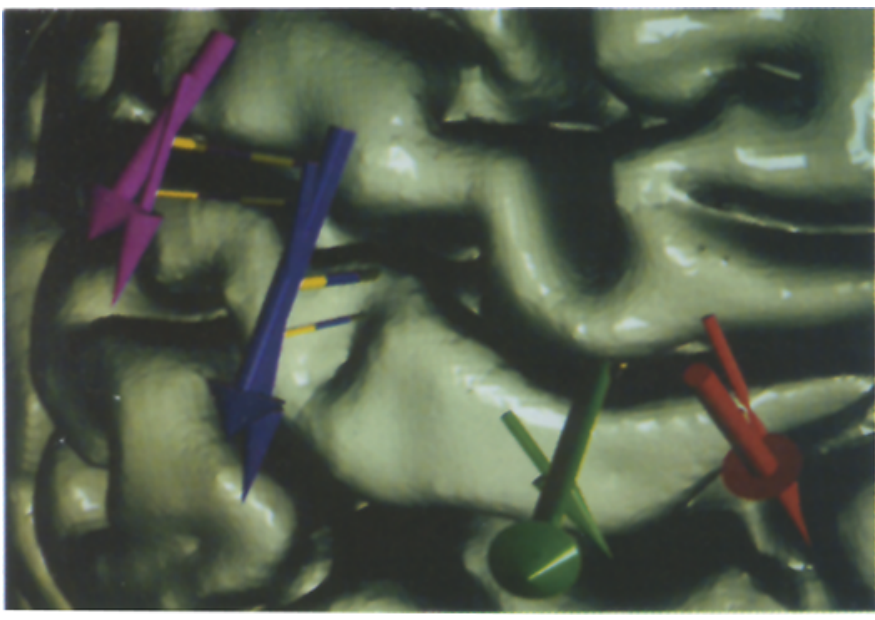

Figure 7. Same as figure 6, except that the results obtained for the model of a dipole patch in a realistic volume conductor are plotted. Heads of thick arrows corresponding to 2 nAm.

the collarbone. Thus, in two cases, the goodness of fit values are better than those obtained with the spherical model; in the other two cases goodness of fit values are worse. Most striking is that all four dipoles estimated using the realistic volume conductor model seem to have a more or less radial orientation. Furthermore, dipoles obtained for the lip (magenta) and the collarbone (red) are not located in the vicinity of the central sulcus. These observations are completely contradictory to those obtained with the spherical model. Figure 6 obviously reveals a serious problem, one requiring careful consideration. Is the assumption of a source with a predominant tangential orientation untenable for SEF? (This assumption is implicitly made by choosing a spherical volume conductor model.) Or was the implementation of the realistic volume conductor model inappropriate?

The answer to these questions is relatively subtle and requires some basic understanding of the nature of inverse problems. To find the key to the correct answer, it is necessary to recall that, in the case of a realistic volume conductor, the magnetic field produced by a dipole oriented perpendicular to the surface ("radially") is generally an order of magnitude smaller than the field produced by a tangentially oriented dipole of the same strength (Menninghaus et al. 1994, Menninghaus and Lütkenhöner 1994). With regard to a parameter optimization procedure this means that one of the six model parameters (three parameters for each the dipole location and the dipole moment) tends to have little effect on the outcome. It is obvious that this goes against the endeavor of the solution algorithm to use all its parameters for an optimal fit of the data. However, since the parameter estimation algorithm is not concerned with physiological plausibility, there is a remedy: Having an unphysiologically high amplitude, a radial dipole can produce magnetic field amplitudes comparable to those experimentally observed. This is exactly the effect seen in figure 6: The parameter optimization algorithm provides the dipole with a radial component being an order of magnitude stronger than the two tangential components.

After having explained the approximately radial orientation of the dipoles estimated with the realistic volume conductor model, it is easy to understand the considerable location differences observed in two of the four cases (magenta and red dipole in figure 6). These location differences merely express the fact that the optimal location for a dipole with two tangential components is not necessarily optimal for a dipole having both tangential and radial components. Obviously, the problems inherent in the estimation of the radial component are passed on to the estimation of the dipole location.

\section{Source analysis with anatomical constraints}

The problems just described will not occur if the model of a single current dipole is replaced by the dipole patch model. As shown in figure 7 , the estimated dipoles are similar to those obtained with the spherical model. Only in one case (green dipole representing the fifth digit) is there still a significant radial component. In all the other cases the dipoles have a more or less tangential orientation. As found with the spherical model, dipole locations follow the course of the central sulcus.

To determine whether the dipoles are located on the anterior wall of the postcentral gyrus (area $3 b$ ) or on the posterior wall of the precentral gyrus, the surroundings of the dipole locations (area $\pi \mathrm{cm}^{2}$ ) were dye-marked using the color of the respective dipole (figure 8). The brain was plotted as semi-transparent, except for the vicinity of the central sulcus. It can be seen that only one dipole (blue arrow obtained for the first digit) is found on the anterior wall of the postcentral sulcus, as expected, whereas the other dipoles are misallocated to the other wall. However, this kind of localization error corresponds to an inaccuracy of only a couple of millimeters. A projection to the correct wall could be forced, for example, by making an additional assumption about the direction of the current dipole (inward or outward with respect to the cortical surface).

\section{Discussion}

The examples demonstrate that replacing the simple model of a homogeneous sphere with a more realistic 


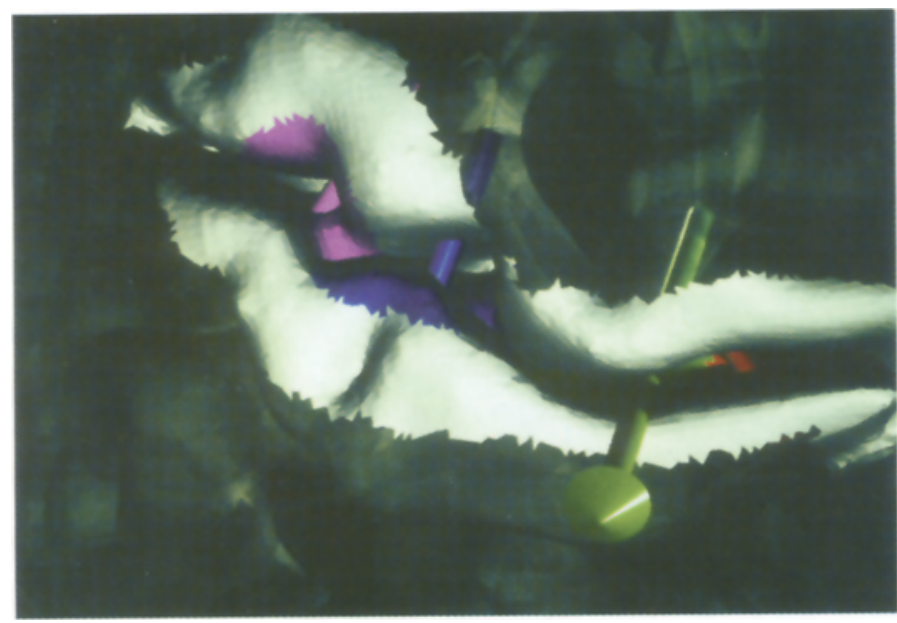

Figure 8. Alternative visualization of the fit result obtained with the model of a dipole patch in a realistic volume conductor (virtual camera at the same position as in figure 7). The dipoles are plotted at their true location, and the surroundings of these locations (area $\mathrm{cm} 2$ ) are dyemarked with the color of the respective dipole symbol. The cortical surface was plotted as semi-transparent, except for the vicinity of the central sulcus.

volume conductor model does not necessarily ensure a more reliable interpretation of the data. On the contrary, it is likely that radial source components are overestimated by an order of magnitude if typical experimental data are analyzed using the model of a current dipole in a realistic volume conductor. Moreover, one runs the risk of ruining estimated source locations, as a consequence of this overestimation.

A basic explanation for the apparent paradox that improving the volume conductor model deteriorates the results was already given above. This problem shall now be approached in a slightly different way. First, the case that the measured data have an almost perfect dipolar character shall be considered. This was the situation, for example, in the experiments of Menninghaus et al.(1994), who studied the magnetic field generated by an artificial current dipole in a phantom of a human skull filled with saline solution. Owing to the fact that a single current dipole was an almost perfect source model and that the signal-to-noise ratio was very good, the localization errors obtained with a realistic volume conductor model were less than $3 \mathrm{~mm}$. An overestimation of the radial dipole component was not observed in that study. The reason is that the match between model and data was so good (goodness of fit about $99.9 \%$ ) that erroneously introducing a significant radial component would have been counter-productive.

However, in a typical experiment (like that considered in this article) the model of a current dipole represents a strong simplification, often an oversimplification. This means that the attainable goodness of fit is not only limited by noise contributions in the measured data (as in the experiments of Menninghaus et al. (1994)), but also by the inappropriateness of the model. Since a parameter optimization algorithm minimizes only a certain figureof-merit function (quantifying the difference between model and measurement), it cannot be expected that the way the model parameters are used is necessarily meaningful from a physiological point of view. Thus, the seeming superiority of the spherical model, as observed in the present study, does not mean that this model is actually a better volume conductor model than the realistic model, it only demonstrates the enormous importance of source model constraints: when working with a spherical volume conductor model, dipole moments are implicitly constrained to a tangential orientation.

There are several ways to eliminate the overestimation of the radial dipole component. Certainly, the best way is through a simultaneous modeling of electrical and magnetic data. This requires, however, that both types of data are available and that an adequate modeling of the intricate distributions of the electrical conductivities (which can be estimated only to a limited extent) exists. If only magnetic data are at hand, it is necessary either to exploit prior knowledge about the sources or to make reasonable assumptions about the solutions. A very simple approach would be to set the radial component to zero (radial and tangential would refer in this case to the best spherical approximation of the realistic volume conductor). A disadvantage of this procedure is that the capability of the realistic model to provide information about the radial dipole component is not used. Another solution would be to use a penalty for high amplitudes of the dipole moment. However, as a consequence of the latter procedure, the estimated dipole depth would be biased, because superficial dipole locations would have an advantage compared to deeper dipole locations. All these disadvantages can be avoided if the dipole patch model is used: no prior assumptions about the amplitudes of the dipole moments are required, and, in principal, the model provides information not only about the tangential dipole components, but about radial ones as well. A closely related model was recently used by Wieringa $(1993$, p. $70 f)$ to estimate the size of the activated cortex area from the strength of the estimated dipole moment.

The question to be discussed now is whether a much simpler model wouldn't be sufficient: a single current dipole riding on the cortical surface, with its moment orientated perpendicular to the surface. To understand why this question must be answered in the negative, it is important to realize that a basic feature of the latter model is a coupling between dipole location and dipole orienta- 
tion, i.e., it is no longer possible to choose these two quantities independently. The following consideration shows the serious consequences of this fact.

A current dipole is usually considered to be an equivalent source. Its location can be interpreted as the "center of gravity" of the real source, and its moment represents the vectorial sum of all the current vectors in the source. However, in the case of a current source distributed on a curved surface (as the cortex), it is a rare exception if the center of gravity is located on the surface. Furthermore, even if the center of gravity were located on the surface, it cannot be expected that the normal direction in that surface point will be identical with the direction of the vectorial sum of all the currents in the source. In practice this problem is aggravated by the fact that only a discretized surface is available, and therefore the direction perpendicular to the surface is severely affected by discretization errors. The dipole patch model, implemented here in one of its most basic forms, removes all of these problems quite naturally. Expressed in physiological terms, the underlying assumptions imply that the level of neural activation is constant throughout the patch. Of course, many other activation patterns could be assumed as well; for example, a pattern corresponding to a two-dimensional Gaussian distribution. Such a shape would provide a smooth transition between activated regions and non-activated regions of the brain.

It is evident that the dipole patch model can replace the simple current dipole model in almost all conceivable applications, including spatiotemporal multiple dipole modeling and analyses with the MUSIC algorithm (Mosher et al. 1992). An exception is the rotating dipole model (fixed location, components of dipole moment independent and time-varying), since the dipole orientation is not an independent quantity in the case of the dipole patch model (however, this not a disadvantage of the dipole patch model, since the idea of a rotating dipole is not very plausible from the physiological point of view). The simultaneous activation of several patches may account for simultaneously active sources. For the analysis of slower SEF components, e.g., one patch might be restricted to movement in SII, while the other could account for activities in SI.

\section{References}

Dale, A.M., and Sereno, M.I. Improved localization of cortical activity by combining EEG and MEG with MRI cortical surface reconstruction: a linear approach. J. Cognit. Neurosci., 1993, 5: 162-176.

Fuchs, M., Wagner, M., Wischmann, H.A., Ottenberg, K., and Dössel, $O$. Possibilities of functional brain imaging using a combination of MEG and MRT. In: C. Pantev, T. Elbert, and B. Lütkenhöner (Eds.), Oscillatory event related brain dynamics. Plenum Press, New York, 1994: 435-457.

Hämäläinen, M.S., and Sarvas, J. Realistic conductivity geometry model of the human head for interpretation of neuromagnetic data. IEEE Trans. Biomed. Eng., 1989, 36: $165-171$.

Hari, R., Joutsiniemi, S.L., Sarvas, J. Saptial resolution of neuromagnetic records: Theoretical calculations in a spherical model. Electroenceph. clin. Neurophysiol., 1988, 71: 6472.

Kuriki, S., Isobe, Y., Mizutani, Y., and Murase, M. Magnetic responses evoked by verbal and nonverbal stimuli. In: $K$. Atsumi, M. Kotani, S. Ueno, T. Katila, and S.J. Williamson (eds.), Biomagnetism '87. Tokyo Denki University Press, Tokyo, 1988: pp. 262-265.

Kuriki, S., Murase, M., Takeuchi, F. Locating accuracy of a current source of neuromagnetic responses: Simulation study for a single current dipole in a spherical conductor. Electroenceph. clin. Neurophysiol., 1989, 73: 499-506.

Lehnertz, K., Lütkenhöner, B., Hoke, M., Pantev, C. Considerations on a spatio-temporal two-dipole model. In: Advances in Biomagnetism (S.J. Williamson, M. Hoke, G. Stroink, M. Kotani, eds.), Plenum Press, New York, 1989: pp. 563-566.

Lütkenhöner, B. A simulation study of the resolving power of the biomagnetic inverse procedure. Clin. Phys. Physiol. Meas., 1991, vol. 12, suppl. A, pp. 73-78.

Lütkenhöner, $B$. On the biomagnetic inverse procedure's capability of separating two current dipoles with priori known locations, in: Biomagnetism: Clinical Aspects (M. Hoke, S.N. Erné, Y.C. Okada, G.L. Romani, eds.), Excerpta Medica, Amsterdam, 1992a: pp. 687-692.

Lütkenhöner, B. Möglichkeiten und Grenzen der neuromagnetischen Quellenanalyse. Münster/Hamburg: Lit, 1992b.

Lütkenhöner, B. Dipole and multidipole source analysis of magnetic fields: Possibilities and limitations. In: Proceedings of the 9th International Conference on Biomagnetism (L. Deecke, C. Baumgartner, eds.), Elsevier, Amsterdam, 1994a (in press).

Lütkenhöner, B. Effect of parameter space reduction on dipole localization accuracy exemplified for a simple model of a sulcus of the brain. In: Proceedings of the 9 th International Conference on Biomagnetism (L. Deecke, C. Baumgartner, eds.), Elsevier, Amsterdam, 1994b (in press).

Lütkenhöner, B., Pantev, C., Hoke, M. Comparison between different methods to approximate an area of the human head by a sphere. In: Auditory evoked magnetic fields and poten-. tials (F. Grandori, M. Hoke, G.L. Romani, eds.), Advances in audiology, vol. 6, Karger, Basel, 1990: pp. 103-118.

Lütkenhöner, B., Lehnertz, K., Hoke, M., Pantev, C. On the biomagnetic inverse problem in the case of multiple dipoles. Acta Oto-Laryngol. (Stockh.), 1991, suppl. 491: 94-105.

Lütkenhöner, B., Pantev, C., Hoke, M. Interpretation of auditory evoked magnetic fields in terms of two simultaneously active dipoles. Biomed. Res., 1992, vol. 13, suppl. 1 , pp. 17-22.

Lütkenhöner, B., and Pantev, C. The exogenous components of the auditory evoked field: current status, in: Biomagnetism: Clinical Aspects (M. Hoke, S.N. Erné, Y.C. Okada, G.L. Romani, eds.), Excerpta Medica, Amsterdam, 1992: pp. 143152.

Meijs, J.W.H., Bosch, C., Peters, M.J., Lopez da Silva, F.H. On 
the magnetic field distribution generated by a dipolar current source situated in a realistically shaped compartment model of the head. Electroenceph. clin. Neurophysiol., 1987, 66: 286-298.

Menninghaus, E., Lütkenhöner, B., and Gonzalez, S.L. Localization of a dipolar source in a skull phantom: Realistic versus spherical model. IEEE Trans. Biomed. Eng., 1994, 41: 986-989.

Menninghaus, E., and Lütkenhöner, B. How silent are deep and radial sources in neuromagnetic measurements. In: Proceedings of the 9 th International Conference on Biomagnetism (L. Deecke, C. Baumgartner, eds.), Elsevier, Amsterdam, 1994 (in press).

Mosher, J.C., Lewis, P.S., and Leahy, R.M. Multiple dipole modeling and localization from spatio-temporal MEG data. IEEE Trans. Biomed. Eng., 1992, 39: 541-557.

Pantev, C., Hoke, M., Lehnertz, K., Lütkenhöner, B., Fahrendorf, G., Stöber, U. Identification of sources of brain neuronal activity with high spatiotemporal resolution through combination of neuromagnetic source localization (NMSL) and magnetic resonance imaging (MRI). Electroenceph. clin. Neurophysiol., 1990, 75: 173-184.

Penfield, W., and Rasmussen, T. The cerebral cortex of man: A clinical study of localization and function. New York: Mac- millan, 1950.

Press, W.H., Teukolsky, S.A., Vetterling, W.T., and Flannery, B.P. Numerical recipes in C. The art of scientific computing. Cambridge Unviversity Press, Cambridge, MA, 1992.

Reite, M., Teale, P., Zimmermann, J., David, K., and Whalen, J. Source location of a $50 \mathrm{msec}$ latency auditory evoked field component. Electroenceph. clin. Neurophysiol., 1988, 70: 490-498.

Steinsträter, Olaf. Rekonstruktion der Kortexoberfläche auf der Basis von Magnetresonanztomographiedaten. Diplomarbeit, University of Münster, 1994.

Tan, S., Roth, B.J., Wikswo, J.P. The magnetic field of cortical current sources: the application of a spatial filtering model to the forward and inverse problems. Electroenceph. clin. Neurophysiol., 1990, 76: 73-85.

Wieringa, H.J. MEG, EEG and the integration with magnetic resonance images. PhD thesis, Enschede, 1993.

Yamamoto, T., Williamson, S.J., Kaufman, L., Nicholson, C., and Llinas, R. Neuromagnetic localization of neuronal activity in the human brain. Proc. Natl. Acad. Sci. USA, 1988, 85: 8732-8736.

Young, C., Wells, D. Ray Tracing Creations. 2nd edition, Waite Group Press, Corte Madera, CA, 1994. 